\title{
VISUAL TOOL FOR WORKLOAD LEVELING USING THE WORK DENSITY METHOD FOR TAKT PLANNING
}

\author{
Vishesh V. Singh ${ }^{1}$, Iris D. Tommelein ${ }^{2}$, and Lian Bardaweel ${ }^{3}$
}

\begin{abstract}
This paper presents a novel interactive Visual Management tool, called ViWoLZo (Visual Workload Leveling and Zoning), that supports takt planning. It is based on the concept of "work density" to depict workloads visually as well as quantitatively so that it can be used to find an optimal workload level and associated work space zoning. ViWoLZo was created in the course of action research on two projects in the San Francisco Bay Area. Because the concept of work density is generic, the tool can be configured to any construction floor plan and process sequence pertaining to any phase of constructioninvolving repetitive or non-repetitive work - to help level the workload based on the selection of zones. In turn, this leveling informs the user's selection of the takt to use in planning the work. This Visual Management tool delivers proof-of-concept that work density is useful in studying workload leveling and zoning alternatives for takt planning. Use of ViWoLZo replaces subjective approaches for deciding how to divide work space into zones. While work density data is not yet readily available, the tool illustrates what value such data can have by supporting a user's what-if analyses to decide if the desired takt can be met, considering different production rates, preferences for zoning of work space, and other work structuring considerations. The paper concludes with suggestions for follow-on research, including recommendations for tool enhancements and integration of additional planning steps.
\end{abstract}

\section{KEYWORDS}

Takt planning, Work Density Method (WDM), Visual Management (VM), work structuring, workload leveling

\section{INTRODUCTION}

Construction projects tend to start with ambition to create a good schedule and focus on delivering quality work but, as that work progresses, delays may accumulate and jeopardize the schedule, thus increasing pressure to expedite downstream activities. To

1 MS Candidate, Engineering and Project Management Program, Civil and Environmental Engrg. Dept., Graduate Researcher, Project Production Systems Laboratory (P2SL), Univ. of California, Berkeley, CA 94720-1712,+1 510 833-1422, visheshvs@,berkeley.edu, orcid.org/0000-0002-6999-9727

2 Professor, Civil and Environmental Engrg. Dept., and Director, Project Production Systems Laboratory (P2SL), Univ. of California, Berkeley, CA 94720-1712, +1 510 643-8678, tommelein@berkeley.edu, orcid.org/0000-0002-9941-6596

3 MS 2018, Engineering and Management Program, Civil and Environmental Engrg. Dept., Graduate Researcher, Project Production Systems Laboratory (P2SL), Univ. of California, Berkeley, CA 947201712, +1 510 833-1422, lian.bar@pihome.ca, orcid.org/0000-0002-6285-5261 
reduce the duration of the remaining schedule while aiming to still meet the target completion date, project teams resort to tactics such as deploying more parallel activities or expediting them by increasing resources. These tactics tend to increase the number of people working in the same space, potentially leading to trade stacking, increasing safety risk, and hampering the quality of construction.

It is common practice for site managers to divide work space so that activities can take place in parallel. Besides allowing for crews to work concurrently yet not be in each other's way, this practice can shorten the construction schedule, help set activity completion goals, and increase productivity (Binninger et al. 2018, Frandson et al. 2015, Yassine et al. 2014). However, in practice the rationale for dividing tends to not be wellarticulated. Without using data, e.g., on the spatial distribution of different types and quantities of work, a large floor plate in a multi-story building may get divided simply, e.g., into quadrants of equal area. Chances are then that the so-obtained divisions will have different amounts of work relative to one another, resulting in unevenness in the production system that may diminish the advantages of using the practice.

Project teams that strategically use takt planning bring a new rationale. Takt planning, like other location-based planning methods, schedules the use of construction work space along with time. Unlike other methods, it does so while structuring the work so that tasks can be completed within the time of a regular beat determined based on customer demand, with trades involved in those tasks moving through space sequentially from zone to zone as in a Parade of Trades (Tommelein et al. 1999) or like wagons of a train. The desire to plan work to a regular beat is challenged by the fact that construction work tends to be non-repetitive and production rates vary by crew size, scope of work to be done in an area, means and methods used, etc. This challenge is addressed by the Work Density Method (WDM) (Tommelein 2020a) or other takt planning methods that help design the project production system to promote a leveled workflow, define clear hand-offs between trades, and use capacity buffers (Dunnebier et al. 2014, Frandson et al. 2015, Haghsheno et al. 2016, Tommelein 2020b) in order to achieve plan reliability.

Takt, situated in the Just-in-Time pillar of the House of Toyota (e.g., Figure 3-3 in Liker 2004), is used to set the pace for a leveled workload so that customer demand will be met. On an assembly line, this pace must therefore be matched by each station along the line, that is, each station's cycle time must be less than the takt.

The depiction of a manufacturing process as a linear sequence of steps is adequate for various modeling purposes. A linear abstraction is useful when studying production in construction - one of many examples is Tommelein's (2020a) study of how to takt the Parade of Trades - but construction processes have spatial characteristics that make it perhaps more necessary and worthwhile than it is in manufacturing to create models with depictions of space in two or more dimensions. Indeed, throughout the ages researchers and practitioners alike have been engaged in developing physical renderings all the way to virtual $\mathrm{n}$-dimensional building information models (nD BIM).

To advance the state of the art of takt planning in construction, we searched for a concept akin to cycle time, recognizing the necessity to augment cycle time with an explicit reference to where work takes place in space. This led us to coin the term "work density" (Tommelein 2017), an expression pertaining to workload per area (with space modeled in two dimensions) as defined later in this paper. Work density indicates the speed at which the work of trades can progress through space and thus serves as a generic metric common to all trades. On the basis of work density, workloads across trades can be leveled by judiciously zoning the work space, which informs the assessment whether 
the desired takt can be achieved. Using the WDM method, the work density (i.e., the time needed to perform a certain scope of work in a given area) is established before zoning is decided. This allows for the exploration of different zoning instances to find a satisfactory solution, possibly even an optimal one for a given set of conditions.

The work density construct can be used in mathematical optimization. Jabbari et al. (2020) developed the so-called Workload Leveling and Zoning (WoLZo) algorithm that, given (1) a work space and the number of areas in which to zone it and (2) a set of process steps each with their work density map, determines the zoning with the lowest possible takt (ignoring buffering to account for process time variation). As formulated, the WoLZo problem is solvable when the problem is small in size (i.e., only a few trades are considered and the work space is to be divided in only a few zones) but as the problem size grows it quickly becomes computationally prohibitive to solve optimally. With this in mind, we here present an expanded problem formulation while recognizing that mathematical optimization may not be within reach.

In order to study how a project production system using workload leveling and zoning may be implemented in practice, we conducted action research on two projects in the San Francisco Bay Area in California. This paper presents the deliverable produced in the course of this research. It is a visual, interactive tool called ViWoLZo (Visual Workload Leveling and Zoning) that informs takt planners of workload levels based on a userselected zoning configuration of the work space and chosen crew sizes. We discuss design choices, provide details of the tool's features, and offer an example based on data collected on one of the projects. The contribution of this paper is the introduction of a novel, interactive tool that helps to shape the user's thinking about possible zones and workload levels, so that they can assess if their plan can meet the desired takt.

The next section of this paper touches on takt planning and Visual Management, as a lead-in to defining the concept of work density. Building on this concept, the paper then presents the ViWoLZo tool to support workload leveling and zoning, with work density serving as the underlying rationale. The tool was prototyped using data from one project and then further developed and tested using data from a second project. The functionality of the tool is explained and its use is illustrated. The discussion section revisits the assumptions made in the course of tool development. The paper concludes with suggestions for follow-on research, including recommendations for tool enhancements and integration of additional planning steps.

\section{BACKGROUND}

\section{Takt Planning ANd Work Structuring}

For clarity and to promote consistency in language use, the following three paragraphs articulate key terms (put in quotes) and include definitions from earlier papers (Frandson et al. 2013, Jabbari et al. 2020, Tommelein 2020a).

"Takt" is the German word for beat, the regularity with which something gets done. By definition, takt is the unit of time within which a product must be produced (supply rate) in order to match the rate at which that product is needed by the customer (demand rate) (after Hopp and Spearman 2011). For a construction project, these hand-offs between supply and demand take place at the completion of a "chunk" of work either between trades succeeding one another in a process, or between different crews within the same trade. These "chunks" are pieces of work of manageable size with handoffs that are to be decided during phase scheduling (Tsao et al. 2004, Tommelein 2017). 
Takt is used to pace the production rate of a process so that the process will meet the demand rate. Processes in a phase are viewed as Parades of Trades, with trades passing through different work spaces at different times as planned, completing work with timely on-takt handoffs. The trade's timely execution of their work to the takt by designated work area avoids trade stacking and helps to adhere to the plan during execution.

The "master schedule" of a construction project may comprise one or multiple phases. A "phase" is defined so as to include the work of all trades who will work on site moreor-less concurrently in a certain "work space." A phase's start and end are delimited by milestones in the master schedule. Phases comprise one or several processes. Each "process" is made up of steps. Each "step" is performed by one trade so that the process sequence of steps forms a Parade of Trades. Trades may be responsible for performing one or multiple steps in a process.

To speed up the process, the production planning team may divide the work area into "zones" (work areas that are mutually exclusive and collectively exhaustive), and develop a plan while ensuring that each trade has their own zone to work in at any time, never stacking them. Zones may differ in size and shape from one another, but any work in them must be completed within the takt of the Parade as it progresses. In general-although not always - steps in each zone will then have less scope for each trade to complete, and thus may require less time for that scope to be completed until the trade is ready to move to the next zone. Less time required means that the process may be able to meet a lower takt. The takting of a process thus depends on how zones are defined. It is furthermore informed by process time variability in the system (also see, e.g., Binninger et al. 2017).

Several dimensions of work structuring were selected to develop the here-presented ViWoLZo tool so that it would support decisionmaking by allowing a user to (1) change zone boundaries and (2) change crew sizes and thereby adjust production rates, thus varying work densities. Working with these specifics, the process requires iteration to produce the desired schedule. In the current implementation of ViWoLZo, the process and its steps are assumed to be fixed (the user cannot add or remove steps).

\section{Visual Management}

Lean is about creating more value with less waste; doing more with less. Applied to sharing information, Visual Management (VM) aims to increase the value of information by delivering it more succinctly and reducing the effort that people have to make to digest it. To add, in the Toyota Production System, principle 7 states "Use visual controls so no problems are hidden" (Liker and Meier 2005 p. 10). VM supports production management by increasing the communication ability of process elements, defined as process transparency (Formoso et al. 2002). Synthesizing and making information from across the production system available to everyone concerned improves process transparency. It is achieved by making process flows visible and comprehensible through various ways of organizational and physical showcasing of information (Tezel et al. 2016, Brady et al. 2018).

To make the abstract concept of work density understood and operable by the user of ViWoLZo, we applied VM. ViWoLZo provides clarity to its user by displaying work density per trade and per zone in the form of a simple depiction of a work area that can easily be manipulated. In particular, it hides the minutiae of tables with quantities and production rates for trades, as will be further explained in this paper. 


\section{WORK DENSITY}

Work density is a relatively-novel mathematical concept first explored in construction takt planning by Dunnebier et al. (2014) and defined by Tommelein (2017) as: "Given a certain work area, work density describes how much time a given trade will require to do their work in that area, based on the product design and the scope of work done by that trade for a given task in the schedule (thus depending on work already in place and work that will follow), the means and methods the trade will use to do their work while accounting for their crews' capabilities and crew size." Thus, for a given scope of work in a certain unit of space and associated work structuring specifics, work density describes the unit of time the trade will need to complete that scope. Being a generic and simple numerical value, work density compiles specifics of several production-system throttles describing what work will be done, by whom, where, and how?

For the ViWoLZo tool presented here, many of these throttles are set. Work density can then be treated as the mathematical product of the quantity of work in a given area and the rate at which a particular crew can perform that work, namely:

$$
\begin{aligned}
& \text { Work Density [time/area] } \\
& \quad=\text { Quantity [units/area] * Production Rate [time/unit] }
\end{aligned}
$$

Work density varies by location based on what work is to be done and where (e.g., install sink 4 in bathroom 3). It is also process-specific, that is, it is expressed in terms of the work that is included in a given step. For example, does the installation step include putting only the sink fixture in place, and is that step followed by a step later in the process that includes mounting the faucet and connecting it to the plumbing system? Alternatively, are both steps combined into one, or is the work perhaps spread out over more than two steps? For each type of work included in a step, a production rate must be defined. Given a certain type of work in a given location, a crew size, and their corresponding production rate, a project-specific work density map is populated. When different types of work are aggregated into one step (e.g., placing the fixture and connecting the plumbing) work density maps are assumed to be additive. If that assumption does not hold, a single production rate for that step may be provided to account for the aggregation of work.

\section{TOOL DESIGN}

\section{Data Collection and Organization}

Takt planning requires up-front effort for data collection. Since the plan pertains to processes, all trades involved in these processes are to participate in the plan's development. Different spaces (e.g., corridor, open space, office) may be "visited" by different trade crews in different process steps. Knowing the production rate for each crew involved in a process step and recognizing the types of work each crew is to perform in different locations are essential in generating the plan.

Meeting with all the trades involved during phase planning allows everyone on the team to develop a shared understanding of the process flow they will follow. The information gathered in these meetings results in:

- Process maps with steps, and crews involved in each step

- Production rates

- Quantity take-offs

- Work density maps

Each of these is expanded on next. 


\section{Process Maps With Steps AND Crews InVOlVed}

Work structuring is project production system design. It answers the questions: "(1) In what units will work be assigned to groups of workers? (2) How will work be sequenced? (3) How will work be released from one group of workers to the next? (4) Will consecutive groups of workers execute work in a continuous flow process, or will their work be decoupled? (5) Where will decoupling buffers be needed and how should they be sized? (Howell et al. 1993) and (6) When will different units of work be done? (Tsao et al. 2004)." With these questions in mind, a project is viewed as made up of different work spaces as per the different types of work and trades related to them, e.g., a floor may comprise corridors, open areas, closed rooms, bathrooms, etc. These work spaces are defined for their unique sequence of steps required to complete the work in that space.

Takt planning has the scheduler collaborate with all trade partners involved in a phase to identify the processes involved and to engage in work structuring. The team must define these processes by describing step-by-step, for each of these different spaces, what work is to be done and in what sequence. Each step will be performed by a crew of one trade partner, based on their designated scope of work and how that work is structured. The identification of processes and spaces tends to require iteration.

A process map visually represents each step during which a trade partner will be performing a specific scope of work. Figure 1 illustrates the process map of a corridor space from the second project we studied. Steps are color-coded to identify which trade partner is in charge, e.g., blue depicts the electrical specialty contractor. Similarly, jointly with the project team we created process maps for several unique spaces identified on a typical floor. The example expanded on in this paper includes the steps in-between the two brown-colored steps done by the drywall trade. While mapping processes, hand-offs must be included at the beginning and the end of the takted process, in order to understand the constraints that affect the start of the process and to understand the needs of the customers at the end of the process. Creating process maps for different project spaces allows the team to explore alternative steps with their sequencing and to identify different crews and their scopes of work in the process. Each trade must then provide production rates and work quantities for the step that is theirs.
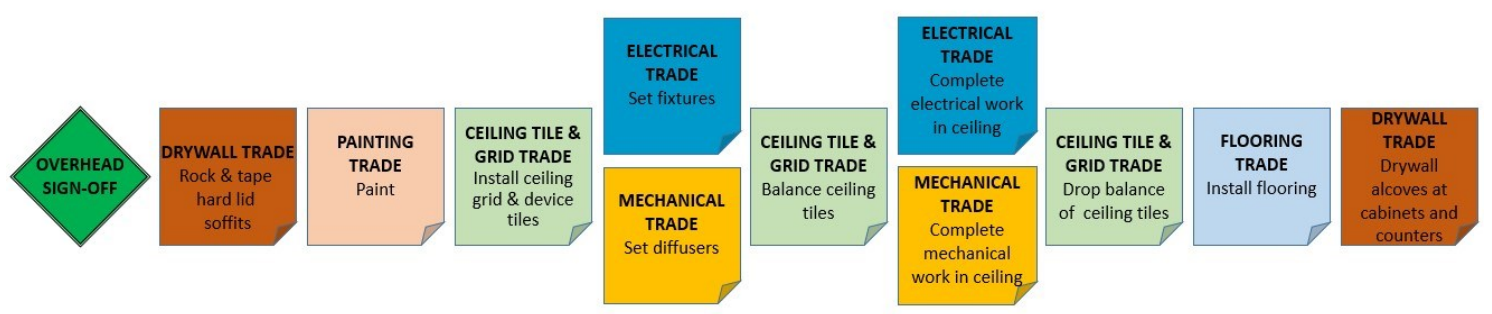

Figure 1: Process Steps for Corridor Work in the Finishing Phase (Luktuke 2019)

\section{Production Rates}

\section{Production Rates and Throttles}

We obtained production rates for each step in each process, associated with different crews for each trade partner. These rates stemmed either from the trade's historical database or were articulated by the foreman or project manager based on their experience. For each step identified in the process map, the rate corresponds to a certain crew size; the default values in ViWoLZo are those provided by the trades. A ViWoLZo user who wants to increase or decrease a workload associated with a step, can alter the size of the 
crew associated with that step so as to change the production rate and hence the work density by using Equation 1. The default assumption is that production rates vary in a linear fashion with crew sizes. Recognizing that several throttles affect the production rate (e.g., means and methods on-site vs. use of pre-fabrication, production sequence, weather conditions) and that these throttles have many alternatives and inter-relationships, the user may prefer to manually adjust production rates.

\section{QUANTITY TAKE-OFFS}

\section{Defining Cells}

The novelty of ViWoLZo lies in its support for users to try different zoning scenarios. Cells are the basic units that make up a zone. The user can choose a single cell or aggregate a combination of cells to form a zone. Cell grids may be defined in different ways. They may be generic or line up where a potential zone boundary might come. The smaller the size of the cells (i.e., the more cells the work space is divided into), the greater will be the number of possibilities for leveling the workload so that a lower takt may be achievable. However, this comes at the cost of increased effort. Having more cells will require greater front-end effort to set up the tool (a one-time cost) and obtain quantity take-offs, and more computation to determine workloads (a recurring cost).

In exploratory research, Bardaweel (2018) defined a work density map using evenlyspaced grid cells. In follow-on research that focused on interior finishes, we wanted to be able to align the grid with architectural features such as walls of rooms or open spaces, so we allowed the grid spacing to vary. Different spaces (e.g., open space, office space, corridors, bathrooms, special rooms, and core wall areas) dictate the border of cells that are parallel and near the intersections of these spaces. Especially when planning interior finishes work, it may be preferable to have cell borders aligned with architectural features so that a cell doesn't have areas from different spaces. However, this is not always preferred. For example, if a wall needs to be worked on from both sides, assigning one of its sides to one cell and the other side to another cell could lead to incorrectly capturing work density. Instead, both walls may have to be included in the same cell.

Spaces are differentiated based on knowledge of construction materials and means and methods, but also as recommended by project personnel involved in the finishing phase. Thus, trade preferences in tackling work for better performance and safety are incorporated (e.g., cells should not be too small in size or hamper any trade's scope). Following discussion with the project team, boundary preferences were incorporated as priority lines on the floor plan. With in-wall inspection being the final customer of the process and the authority to identify priority walls, it was essential to involve personnel responsible for inspections in deciding cell boundaries. Using these priority lines, cell grids were generated. With unit cells defined, work density calculations then required the production rate and product quantities tied to each cell for each step in the process. Depending on the phase of the project, the people involved and the rationale for defining cells will vary.

\section{Capacity Buffer}

Takt planning, in contrast with other space scheduling methods, uses capacity buffers to deal with process time variation. For example, production units may be under-loaded by assigning them work up to 70 or $80 \%$ of their capacity (Frandson et al. 2015). The determination of capacity buffers needed by each trade is given consideration during the workload leveling process (described later). At this stage of defining ViWoLZo data, 
recognizing that capacity buffers will be needed and added later in the planning process, production rates were taken on the lower side of the estimate and rounded conservatively.

\section{Quantity Take-Offs}

Using the Bluebeam Revu software, Bardaweel (2018) collected quantity take-offs and transferred them to Microsoft Excel to calculate work density. Because Bluebeam does not have any feature to allocated take-off quantities to cell by cell, these take-offs had to be done individually for each cell and for each trade.

When working with many different quantities and a floor plan divided into many cells, getting accurate data related to each small cell on the grid is tedious and time-consuming. Microsoft Excel 365 (version 1904) and the take-off program On-Screen Takeoff 3 (OST) (version 03.95.02.51) helped to automate this process. OST allows the user to define each cell (the OST program calls these "zones," but these are not to be confused with our use of the term "zone" in takt planning, where a single cell or a combination of cells form a takt zone). With the manual input of take-offs on OST and then copying the data to Excel, the tool identifies the cell grid name, the process steps active in that cell with their quantities, and automatically populates the quantity maps.

Existing Building Information Modeling (BIM) tools do not appear to have the sought-after functionality. Further research is needed to develop tools that can extract the requisite quantity data from BIM at the level of detail of cells.

\section{WORK DENSITY MAPS}

A work density map for a given process step and trade crew represents how work is spread across a floor. With steps defined during work structuring, production rates collected from trades, and quantity take-off maps generated as per the cell grid established, ViWoLZo computes work density maps cell by cell, simply by using Equation 1 .

ViWoLZo creates a work density map for each crew corresponding to each step. When there is a possibility of combining multiple crews (usually of the same trade) that can/will work together as a team in the same zone and step, their individual work density maps can be added to generate an aggregate work density map. For example, door frame and door panel can be installed separately or together. In our second project, one space had the door panel to be installed separately. Another space, because of different flooring material, had the door panel to be installed along with the frame, so the work density maps for these steps were added together. This step (keeping the steps separate but combining their work density maps into one map) is appropriate because these crews will have a different type of work and thus different work quantities and production rates. Work density maps feed directly into the dashboard of the tool.

\section{APPLICATION}

\section{Project Context}

We started by studying a multi-story medical office building located in the San Francisco Bay Area and selected repetitive floors 2 to 5 to prototype our first workload leveling and zoning interface (Bardaweel 2018). The focus was on takting the process involving interior trades, namely those working on overhead systems, interior walls, in-wall plumbing and electrical, framing of doors, drywalling, and painting.

We then reprogrammed and expanded this prototype in follow-on research (Singh 2019). The second study involved a mixed-use building located in San Francisco, California. This facility has a gross area of $31,500 \mathrm{~m}^{2}\left(340,000 \mathrm{ft}^{2}\right)$ and houses spaces for 
teaching, research laboratories, conference rooms, administration offices, and clinics. The building had to meet a high standard of quality, which required strict inspections. The building is divided into: (1) a 3-story north wing, (2) a 12-story tower, and (3) a 5-story south wing. We focused on the tower with repetitive floors 6 to 12 and, as in the first study, modeled the process involving interior trades. We selected a typical floor among those 7 floors in the tower and created a so-called zoning dashboard for ViWoLZo. The same research approach would apply to other phases.

\section{ZONING DASHBOARD}

The dashboard acts as a visual control for the purpose of defining zones. It is divided into three parts as shown in Figures 2 and 3. Figure 2 (on the left) shows the floor plan with zoning and (on the right) the zoning input tool. Figure 3 offers a visualization of levels of work densities by trade and aggregated by zone, (on the left) before- and (on the right) after balancing workloads.
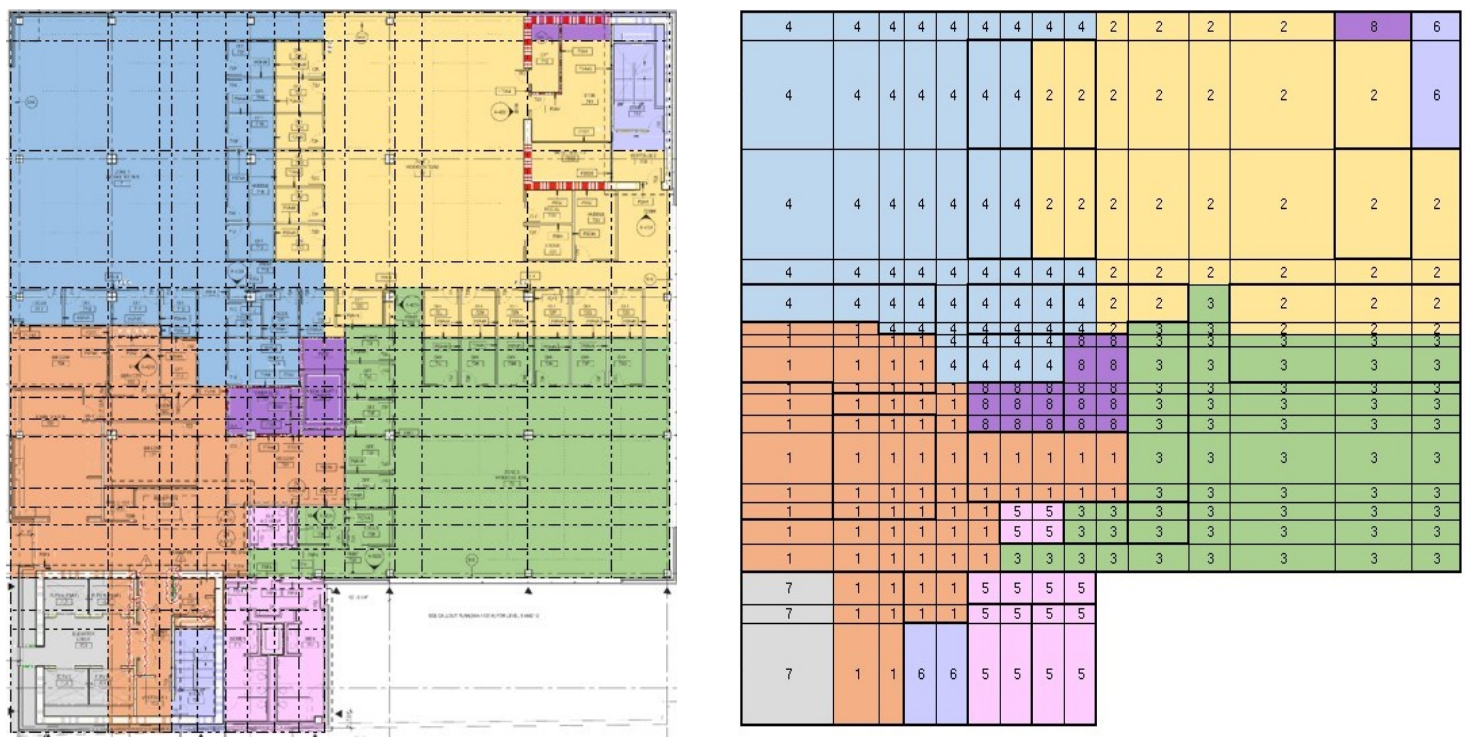

Figure 2: Floor Plan with Zoning (left) and Zoning Input Tool (right) of the Dashboard showing Zoning after Leveling (Figure 3 right)
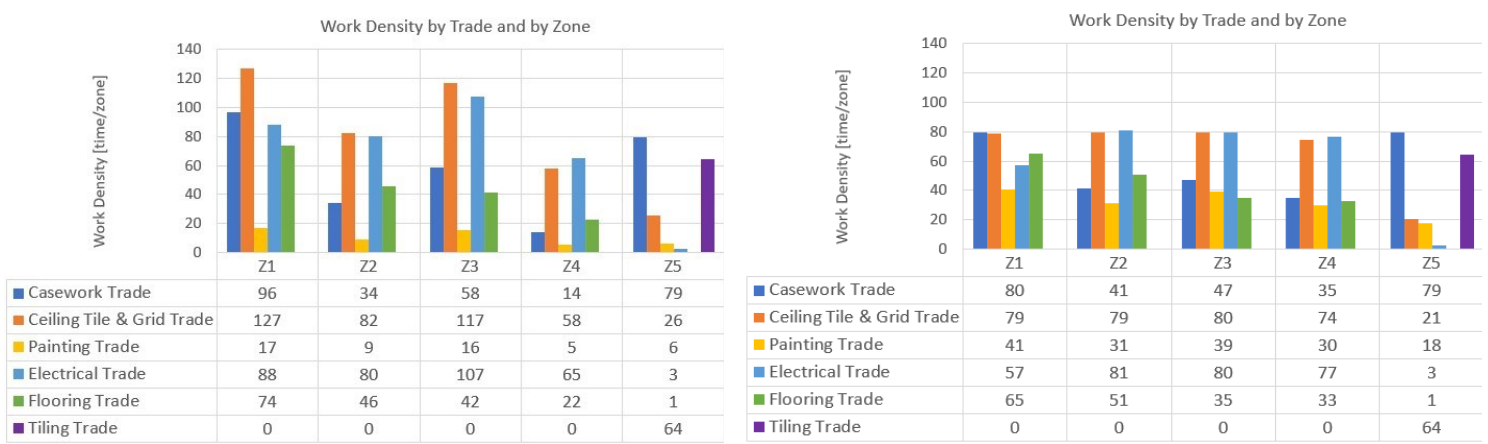

Figure 3: Zoning Dashboard for Work Density Visualization: Before (left) and After (right)

The zoning input tool allows the user to assign a number (each one assigned to a unique color) to each cell to designate which zone it belongs to. The tool then filters all work 
density maps (underlying the dashboard) based on this zoning (Figure 2), adds up the work densities in the cells in those zones, and plots the workload histogram (Figure 3).

By construction of the data that serves as input to ViWoLZo, the number of work density values to be visualized can be quite large. In order to ease the user's interpretation of the data while leveling work densities, the tool combines work density maps of each crew/step at a higher level of abstraction, e.g., by trade. When the user achieves the output they desire to visualize at this higher level, they can hone in on a specific process and fine tune the workload leveling within that process. The histogram then represents work densities for the different steps for the selected process, aggregated by zone.

ViWoLZo is configured to have more zone numbers to select from, than the maximum number of trades working in the work area (floor). This allows for numbers to designate cells with work that is not included in the process. E.g., in Figure 2 (left and right), zone number 6 refers to the North and South stairs, 7 to the elevator core, and 8 to the electrical room, mechanical shaft, and telecommunication distribution room. These zones may be built according to their own schedule, in a process that is takted or not. While leveling the workloads of zones 1 to 5, the user will simply ignore the histogram bars of zones 6 to 8 .

By changing the numbers in cells and the number of zones, the ViWoLZo user can level the workload of each trade within each zone. Leveling is likely to require several iterations of trial and error. The real-time visual representation of work densities will indicate if a better zoning map may be possible. Because zoning is done manually, the user can incorporate site-specific constraints and reflect their preferences in zoning.

Initially, the project team had divided the floor into four quadrants $(Z 1-Z 4)$ plus one zone for bathroom spaces (Z5), thus obtaining the histogram shown in Figure 3 (left). Using ViWoLZo, by renumbering 46 cells in order to change the zone to which they belonged, while considering site constraints and changing crew sizes by adding 7 and removing 4 members, they obtained the zoning shown in Figure 2 (left and right) with the more balanced histogram shown in Figure 3 (right).

Workload leveling is a research topic on its own. It may be done in several ways depending on the work densities shown by bars in the histogram (Figure 3). For example, where the work density is low, the bar can possibly be raised by increasing the number of cells included in that zone or by reducing the production rate by downsizing the associated crew (Eq. 1). Similarly, where the work density is high, the bar can possibly be lowered by reducing the number of cells included in that zone or by throttling up the production rate. If one trade's work density peaks above all others', the bar may be split in two (or more) so as to give that trade more than one takt to complete their work. These are but a few of the work structuring decisions to be made in the course of takt planning at the operational level. These means of work structuring align with Haghsheno et al.'s (2016) harmonization operations, e.g., (1) changing the time required by altering the number of workers, (2) combining several steps into a single one, and (3) changing/ optimizing/replacing products and processes. Allowing for iterations of trial and error in leveling, within and in-between the trade- and process levels, ViWoLZo's real-time visual depiction of work densities helps users make data-driven zoning decisions.

\section{DISCUSSION AND CONCLUSIONS}

This paper presented the ViWoLZo tool that uses work density for work space zoning and workload leveling. It helps users determine if a process can be takted to satisfactorily meet the customer's demand. ViWoLZo's development involved action research on two 
projects where data was collected and the tool was prototyped and tested. Research remains to be done to assess ViWoLZo's usability in practice.

The process of collecting work density data also needs streamlining. Trades should be prepared and possibly incentivized to participate in this process, open to sharing their production data from past projects while assessing challenges and opportunities presented on the new project. We recommend that early collaboration and data collection be managed by a person internal to the project team (e.g., the superintendent) and also engage the person who manages the schedule, rather than rely on researchers external to the project team. The collaboration should include a discussion of trade-offs to be made in the schedule, buffering to accommodate uncertainties, etc. so that everyone on the team gains in-depth understanding of the data and buys into the resulting takt plan.

While ViWoLZo's creation from scratch required a lot of work and data entry was tedious, once the framework was prototyped and populated, the process of creating and visually representing work density maps was straightforward. Using VM, ViWoLZo's dashboard allows the user to visually explore alternative scenarios of zoning, crew sizing, and production rates for multiple trades and decide on a zone map, without having to go back to underlying data. In this way, ViWoLZo supports what-if analyses so the user can decide if the desired takt can be met. While the current interface supports the manual exploration of alternatives, follow-on research is investigating greater use of BIM to collect work quantities, as well as link ViWoLZo to automation and optimization methods.

\section{ACKNOWLEDGMENTS}

The action research and creation of the ViWoLZo tool described in this paper was supported in part by members of the Project Production Systems Laboratory (P2SL) at UC Berkeley and in part by the National Science Foundation (NSF) under grant number CMMI-1563511. Special thanks are due to the project teams including UCSF staff, Webcor Builders' B33 project team, Turner Construction's Berkeley Way West project team, Ghafari's vPlanner staff, and all trade partners who provided information and shared their thoughts with the researchers. Any opinions, findings and conclusions or recommendations expressed in this paper are those of the authors and do not necessarily reflect the views of the members of P2SL, NSF, or any project team members.

\section{REFERENCES}

Bardaweel, L. 2018. "Exploring the Work Density Method as a Means for Takt Time Planning through a Case Study Example." Indep. Study Report (unpublished), Engineering and Project Management Program, UC Berkeley, CA, 49 pp.

Binninger, M., Dlouhy, J., and Haghsheno, S. 2017. "Technical Takt Planning and Takt Control in Construction." Proc. 25th Ann. Conf. Int. Group for Lean Constr., Heraklion, Greece, 9-12 July, pp. 605-612.

Binninger, M., Dlouhy, J., Müller, M., Schattmann, M., and Haghsheno, S. 2018. "Short Takt Time in Construction - a Practical Study." Proc. 26 th Ann. Conf. Int. Group for Lean Constr., Chennai, India, 18-20 July, pp. 1133-1143.

Binninger, M., Dlouhy, J., Oprach, S. and Haghsheno, S. 2016. "Methods for Production Leveling - Transfer from Lean Production to Lean Construction." Proc. $24^{\text {th }}$ Ann. Conf. Int. Group for Lean Constr., Boston, MA, USA, 20-22 July.

Brady, D.A., Tzortzopoulos, P., Rooke, J., Formoso, C.T., and Tezel, A. 2018. "Improving Transparency in Construction Management: a Visual Planning and Control Model.” Engin., Constr., and Arch. Manage., 25 (1) 1277-1297. 
Dunnebier, D., Cleary, J., Galvez, M., Mizell, C., Mueller, K., Pease, J., and Tommelein, I.D. 2014. Presentation: "An Experiment in Takt Time." Proc. 16 ${ }^{\text {th }}$ Ann. Lean Construction Congress, San Francisco, CA, Oct. 7, pp. 1-26, https://www.leanconstruction.org/media/docs/congress/2014/H5B_LCI2014\%20Pre sentation\%20Draft_Takt\%20Time.pdf, visited on 18 Dec. 2019.

Formoso, C.T., Santos, A.D., and Powell, J. 2002. "An Exploratory Study on the Applicability of Process Transparency in Construction Sites." J. Constr. Res., 3(1) 35-54. Frandson, A., Berghede, K., and Tommelein, I.D. 2013. "Takt Time Planning for Construction of Exterior Cladding." Proc. $21^{\text {st }}$ Ann. Conf. Int. Group for Lean Constr., Fortaleza, Brazil, 31 July-2 August, pp. 527-536.

Frandson, A.G., Seppänen, O., and Tommelein, I.D. 2015. "Comparison between location-based management and takt time planning." Proc. $23^{\text {rd }}$ Ann. Conf. Int. Group for Lean Constr., 28-31 July, Perth, Australia, pp. 3-12.

Haghsheno, S., Binninger, M., Dlouhy, J., and Sterlike, S. 2016. "History and Theoretical Foundations of Takt Planning and Takt Control." Proc. 24 ${ }^{\text {th }}$ Ann. Conf. Int. Group for Lean Constr., Boston, MA, USA, 20-22 July.

Hopp, W.J., and Spearman, M.L. 2011. Factory Physics. $3^{\text {rd }}$ Ed., Long Grove, IL: Waveland Press, Inc., 720 pp.

Howell, G., Laufer, A., and Ballard, G. 1993. "Interaction between subcycles: One key to improved methods.' ASCE, J. Constr. Eng. and Mgmt., 119(4), 714-728.

Jabbari, A., Tommelein, I.D., and Kaminsky, P.M. 2020. "Workload Leveling based on Work Space Zoning for Takt Planning." Automation in Constr., Vol. 118, doi.org/10.1016/j.autcon.2020.103223.

Liker, J. 2004. The Toyota Way: 14 Management Principles from the World's Greatest Manufacturer. New York, NY: McGraw-Hill Education, 330 pp.

Liker, J. and Meier, D. 2005. The Toyota Way Fieldbook, NY, NY: McGraw Hill, 288 pp.

Luktuke, Y. 2019. "Experimenting in Development and Use of Takt Planning: A Case Study on the Block 33 Project, Mission Bay." Indep. Study Report (unpublished), Engineering and Project Management Program, UC Berkeley, CA, June, 50 pp.

Singh, V.V. 2019. "Development of Visual Tools for Takt Time Planning Using Work Density Method." Indep. Study Report (unpublished), Engineering and Project Management Program, UC Berkeley, CA, June, 41 pp.

Tezel, A., Koskela, L., and Tzortzopoulos, P. 2016. "Visual Management in Production Management: a Literature Synthesis." J. Manuf. Technol. Manage., 27 (6) 766-799.

Tommelein, I.D. 2017. "Collaborative Takt Time Planning of Non-Repetitive Work." Proc. 25 $5^{\text {th }}$ Ann. Conf. Int. Group for Lean Constr., Heraklion, Greece, pp. 745-752.

Tommelein, I.D. 2020a. "Work Density Method to Support Takt Planning of Nonrepetitive Construction Work.” ASCE, J. Constr. Eng. Manage., in review.

Tommelein, I.D. 2020b. "Takting the Parade of Trades: Use of Capacity Buffers to Gain Work Flow Reliability." Tommelein, I.D. and Daniel, E. (eds.). Proc. $28^{\text {th }}$ Ann. Conf. Int. Group for Lean Constr., Berkeley, CA, doi.org/10.24928/2020/076.

Tommelein, I.D., Riley, D., and Howell, G.A. 1999. "Parade Game: Impact of Workflow Variability on Trade Performance." ASCE, J. Constr. Eng. Manage., 125 (5) 304-310.

Tsao, C.C.Y., Tommelein, I.D., Swanlund, E., and Howell, G.A. 2004. "Work Structuring to Achieve Integrated Product-Process Design.” J. Constr. Eng. Manage., 130 (6) 780-789.

Yassine, T., Bacha, M.B.S., Fayek, F., and Hamzeh, F. 2014. "Implementing Takt-Time Planning in Construction to Improve Work Flow." Proc. $22^{\text {nd }}$ Ann. Conf. Int. Group for Lean Constr., Oslo, Norway, 25-27 June, pp. 787-798. 